\title{
Analysing VLBI images of astrophysical jets via cross-entropy global optimisation method
}

\section{Anderson Caproni ${ }^{1}$, Hektor Monteiro ${ }^{2}$ and Zulema Abraham ${ }^{3}$}

${ }^{1}$ Núcleo de Astrofísica Teórica, Universidade Cruzeiro do Sul, R. Galvão Bueno 868, Liberdade, 01506-000, São Paulo, SP, Brazil. email: anderson.caproni@cruzeirodosul.edu.br

${ }^{2}$ UNIFEI, Instituto de Ciências Exatas, Universidade Federal de Itajubá, Av. BPS 1303, Pinheirinho, 37500-903, Itajubá, MG, Brazil

${ }^{3}$ Instituto de Astronomia, Geofísica e Ciências Atmosféricas, Universidade de São Paulo, R. do Matão 1226, Cidade Universitária, 05508-900, São Paulo, SP, Brazil

\begin{abstract}
Most of jets detected in AGN blazar sources exhibit a morphological structure usually composed by a spatially unresolved core and jet knots receding from it at relativistic velocities. In some cases, the trajectories of the jet components on the plane of the sky seem to be bent, indicating the existence of some kind of acceleration in the respective motion. However, such claims depend strongly on the correct determination of the structural parameters of the jet components, usually obtained from model fitting procedures performed either in the $(u, v)$ or in the image planes. In this work we introduce a new model fitting technique to obtain structural parameters of knots present in VLBI jet images. Our method that is based on the cross-entropy technique minimises an performance function that depends on the sum of the squared residuals obtained from the comparison of an VLBI image and a model image, constructed by summing $N_{\mathrm{s}}$ elliptical Gaussian synthetic sources. We present in this work the cross-entropy model fittings of benchmark images that were built to simulate most of the conditions encountered in typical VLBI images of active galactic nuclei. Besides recovering the parameters of the jet components in all validation tests, our method is able to point out quantitatively the number of the sources present in the image.
\end{abstract}

Keywords. Methods: data analysis, techniques: interferometric, galaxies: jets, galaxies: active.

The determination of the structural parameters of AGN jet components detected from VLBI experiments is crucial to study jet kinematic, as well as to understand the underlying physics ruling such motions. The model fitting parameters of the jet knots are usually obtained from some maximum-likelihood estimator based on gradient techniques. However, the convergence of such algorithms usually depend strongly on the initial guess of the parameters and they are prone to find non-global minimum solutions, especially in the case of too complex images. To cope with those issues, we have developed an alternative method to obtain model fittings to interferometric radio images of astrophysical jets using a global optimisation algorithm known as cross-entropy. This statistical method was introduced by Rubinstein (1997), and it has been used in astrophysics in two different contexts: precession of relativistic jets (Caproni et al. 2009) and isochrones of Galactic open clusters (Monteiro et al. 2010).

Assuming that jet components can be modelled by $N_{\mathrm{s}}$ elliptical Gaussian functions characterised by six parameters (two-dimensional peak position in the image, peak intensity, eccentricity, amplitude and orientation angle of the major axis), our cross-entropy optimisation technique starts generating randomly a set of $N$ individual tentative solutions composed by $6 N_{\mathrm{s}}$ parameters. Each tentative solution generates an image that is compared quantitatively with the observed image through an objective function that depends on the sum of the squared residuals obtained from the difference between them. 

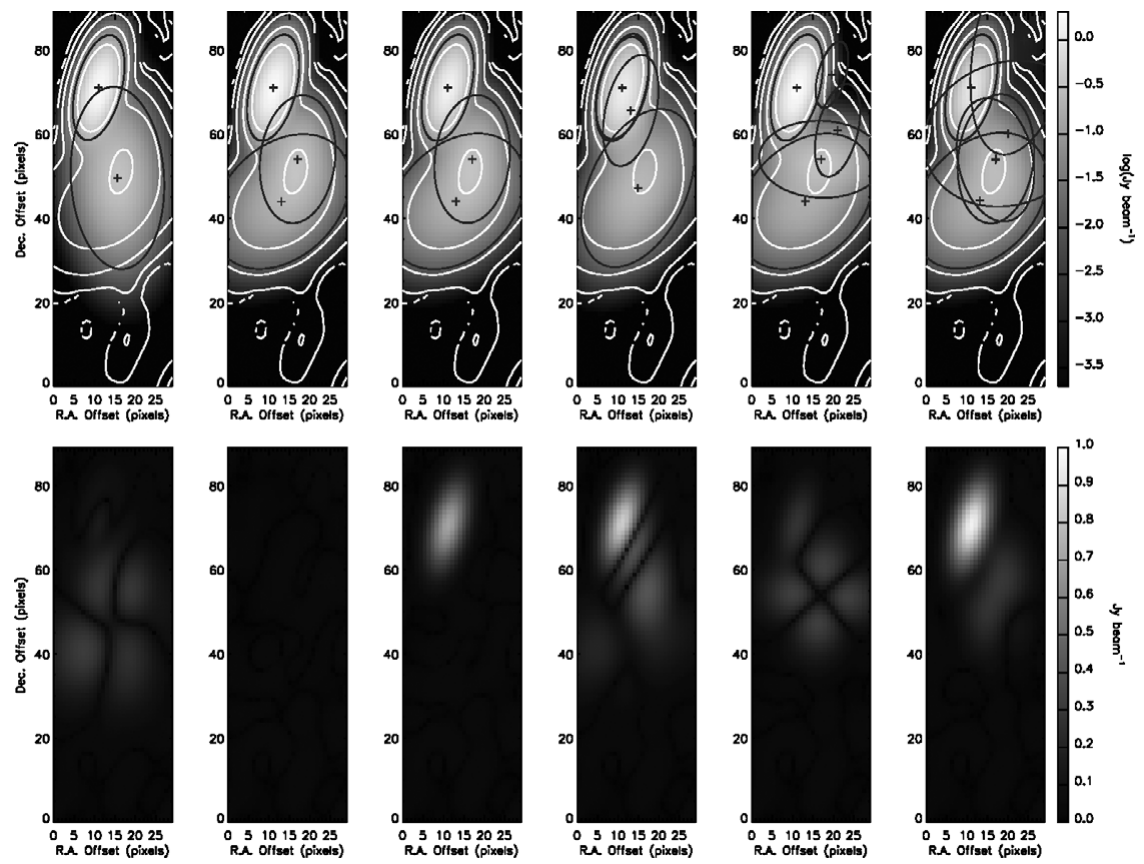

Figure 1. Results of model fitting for a synthetic image composed by 3 elliptical Gaussian sources superposed to a noise background extracted from an image obtained by the MOJAVE project. From the left to right, the number of the sources was varied from 2 to 7 . Top: The contour lines are of the benchmark image, the grey-scale image is constructed from the fitted source parameters and the dark ellipses are the contours of the individual fitted sources (respective centres marked with crosses) at the FWHM. Bottom: The respective residual maps obtained from the absolute difference between the noise and the optimised model images (in linear scale).

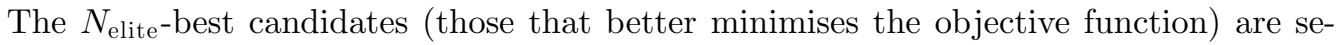
lected and used to construct the next set of tentative solutions. The optimisation process is halted after a pre-specified stopping criterion is fulfilled.

To validate our model fitting optimised procedure, we built benchmark tests that employed synthetic images created to simulate as realistic as possible typical interferometric radio maps. Those images are analysed by our model-fitting technique in order to check its capability of recovering the original parameters of the Gaussian sources. We show in Fig. 1 the model-fitting results from one of this benchmark test. It can also be noted a minimum in the distribution of the residuals for $N_{\mathrm{s}}=3$, as expected in this particular case. A more complete discussion of the benchmark tests and their consequences will be presented in Caproni et al. (2011).

The authors acknowledge the Brazilian agencies FAPESP (Procs. 2010/090006-3 and 2006/57824-1) and CNPq for financial support. This research has made use of data from the MOJAVE database that is maintained by the MOJAVE team (Lister et al., 2009, AJ, 137, 3718).

\section{References}

Caproni, A., Monteiro, H., \& Abraham, Z. 2009, MNRAS, 399, 1415

Caproni, A., Monteiro, H., \& Abraham, Z. 2011, in preparation

Monteiro, H., Dias, W. S., \& Caetano, T. C. 2010, A\& A, 516, 2

Rubinstein, R. Y. 1997, European Journal of Operational Research, 99, 89 